\title{
Chapter 1 \\ From Generic to Specific Musculoskeletal Simulations using an Ontology-based Modeling Pipeline
}

\author{
A. Dicko ${ }^{1}$ and B. Gilles ${ }^{2}$ and F. Faure ${ }^{1}$ and O. Palombi ${ }^{1,3}$ \\ ${ }^{1}$ LJK (CNRS-UJF-INPG-UPMF), INRIA, Grenoble, France ${ }^{2}$ LIRMM \\ (CNRS-UM2), INRIA, Montpellier, France ${ }^{3}$ LADAF, Grenoble, France
}

\begin{abstract}
We present a novel pipeline for the construction of biomechanical simulations by combining generic anatomical knowledge with specific data. Based on functional descriptors supplied by the user, the list of the involved anatomical entities (currently bones and muscles) is generated using formal knowledge stored in ontologies, as well as a physical model based on reference geometry and mechanical parameters. This simulation-ready model can then be registered to subject-specific geometry to perform customized simulations.

The user can provide additional specific geometry, such as a simulation mesh, to assemble with the reference geometry. Subject-specific information can also be used to individualize each functional model. The model can then be visualized and animated.

This pipeline dramatically eases the creation of biomechanical models. We detail an example of a musculoskeletal simulation of knee flexion and hip flexion and abduction, based on rigid bones and the Hill muscle model, with subject-specific 3D meshes non-rigidly attached to the simulated bones.
\end{abstract}

\subsection{Introduction}

The simulation of the human musculoskeletal system is ubiquitous in Biomechanics, Computer Aided Medicine and Computer Graphics. However, many of the models used for these purposes are relatively simple and generic due to the difficulties inherent in their construction. One of the biggest difficulties in the setup of a simulation is to select the relevant anatomical entities: Various bones, muscles, ligaments and organs may have an important role, depending on the phenomenon we want to simulate. This is further complicated by the fact that a plethora of physical parameters are required for correct nu- 
merical simulation. Moreover, it may be necessary to incorporate additional, subject-specific data.

The work presented here is initially motivated by the need to simulate the interaction of medical devices such as braces with specific human bodies, in order to speed up the design process. Another example is to study how a hip or knee prosthesis interacts with individuals with various morphologies during flexion/extension of the knee or abduction/adduction of the hip. The first step of this approach is to create a biomechanical model of the subject. However, this is a very time-consuming and error-prone task, generally difficult to achieve for a mechanical engineer.

In this paper, we introduce a novel pipeline for tackling this challenge. Our pipeline allows a user to select a set of desired functions to simulate and from this functional description a list of approriate anatomical entities can be constructed. This construction is automated using formal knowledge structured in ontologies. The list of anatomical entities involved in the given function is generated, and a physical model based on reference geometry and mechanical parameters is created. This mechanical model may then be personalized using subject-specific parameters, and registered to subject-specific geometric data such as volumetric images, to create a subject-specific model of a given anatomical function. The simulation of arbitrary biomechanical phenomena being beyond the scope of this work, we focus on producing computer simulations of standard tasks such as walking or sitting. However, the functional nature of our modeling pipeline permits extension to a much wider range of scenarios.

Our pipeline, illustrated in Figure 1.1, is targeted to non-specialists as well as expert users. We show how to generate simulations using standard open source simulation software, and to obtain visually pleasing results. The remainder of the paper is organized as follows. Section 1.2 briefly reviews previous work. The anatomical knowledge base and the modeling pipeline are presented in Section 1.3 A simulation is presented in Section 1.4, and results are finally shown and discussed in Section 1.5

\subsection{Related work}

The modeling and simulation of the human body has been a subject of much interest. This attention has produced a cornucopia of methods which are as diverse as the subject areas from which they arise: biomechanics, ergonomics, diagnosis, treatment planning, visualization, graphics, robotics, etc. Consequently, a wide variety of models have been proposed which we briefly review below.

Symbolic models: The most generic models used to describe the anatomy are ontologies. Ontology can be defined as a structured specification of the concepts and semantic, intelligent relations in a field DMT07]. Guttierrez 


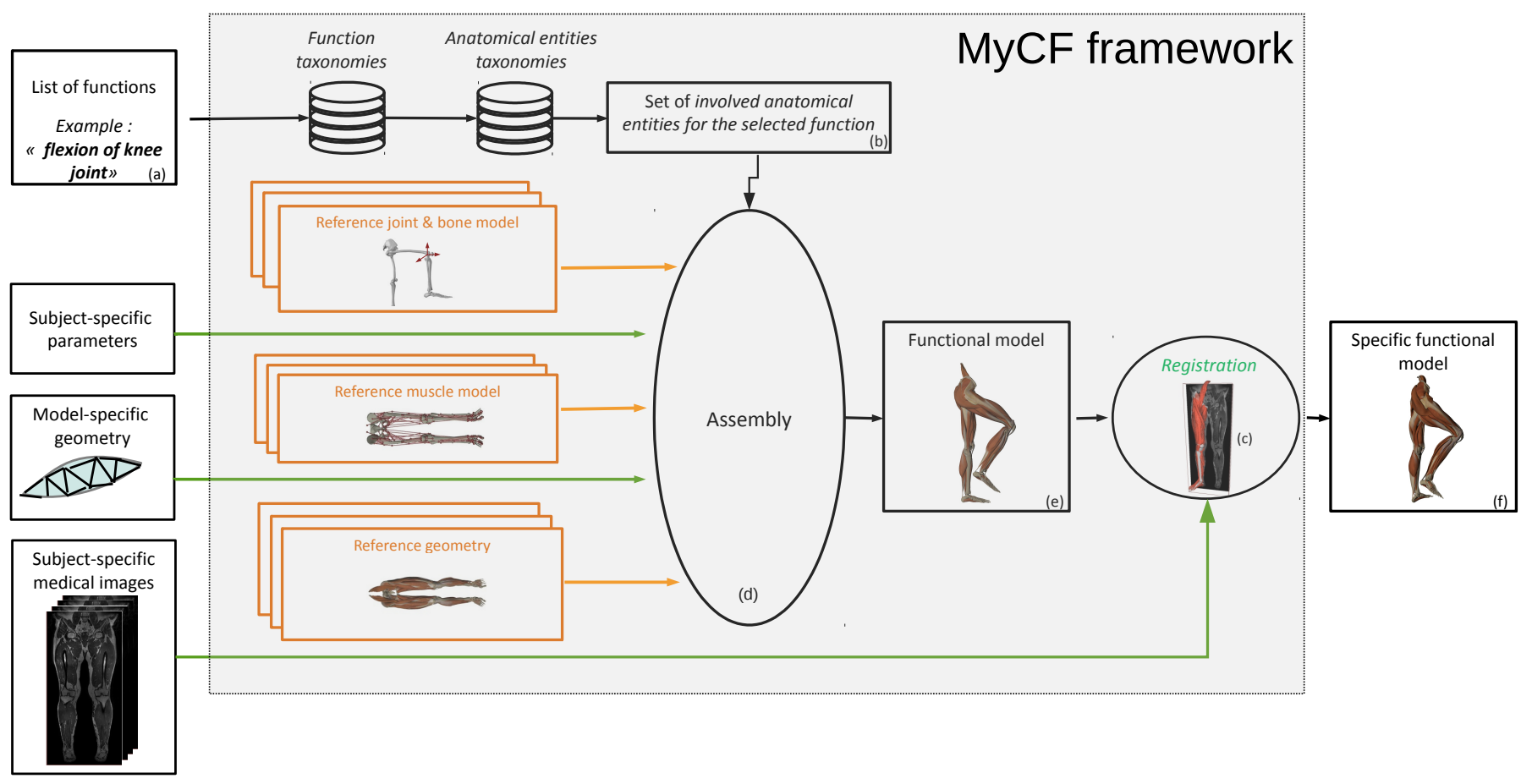

Fig. 1.1 An overview of our modeling framework. On the left, the user input is a list of functions to simulate, optionally complemented with specific data. On the right, the output is a mechanical model ready for simulation. The modeling pipeline uses symbolic knowledge to select anatomical entities to assemble. The final model can be composed of a mix of reference and specific parameters and geometry.

et. al. proposed an ontology of the human shape $\mathrm{GGRT}^{+} 07$, focusing on its relations with the environment.

The anatomical knowledge is the network of relations between entities. An anatomical ontology is a set of taxonomies where anatomical entities are organized as a formal vocabulary. It is organized in data structures easily processed by computers. This data structure contains a list of terms such as femur, psoas, and a list of symbolic relations between this terms describing inheritance or inclusion. The Foundational Model of Anatomy (FMA) RM03 is a widely accepted ontology of the human anatomy and is generally used to denote and navigate across anatomical structures.

One-dimensional representations: The earliest musculoskeletal mathematical model was suggested by Gasser and Hill in 1924 [HG24] to capture global muscle mechanical properties. This model was used to describe the macroscopic relationships between muscle actuation, force-length and veloc- 
ity Hil38], along the muscle path. It was improved by Zajac Zaj89 to a dimensionless aggregate model which can be scaled to approximate subjectspecific musculo-tendon units. The force components are modeled from the measurement of isolated muscle fibers, which directly reflect the non-linear properties due to the sliding filaments. While the series elastic elements can be grouped with the tendon and removed from the model. Pennation effects are directly included into the model Lee10. Schutte improved Zajac's model and added a damping parameter into the force-velocity relationship [Sch93]. Thelen The03 adjusted the parameters to account for changes in muscle properties that occur with aging. Physical models based on a finer discretization, allowing non uniform deformation along the fiber, have been recently proposed [SKP08].

In computer animation and robotics, skeletons are traditionally simplified into acyclic graphs, called animation skeletons or stick figures, composed of one-dimensional segments (limbs) connected by joints. Skeleton poses are then compactly expressed as a set of rotations along the most relevant joint degrees of freedom (i.e., reduced coordinates), which can be easily manipulated or animated based on real data such as motion capture.

Surfacic representations: For visual applications, additional realism can be achieved using a boundary discretization of organs. Procedural methods can then be used to derive organ motion from the pose of an underlying animation skeleton (i.e. Skeleton subspace deformation, also knows as vertex blending or skinning [MTLT88, $\mathrm{KCvO07]).} \mathrm{Although} \mathrm{interior} \mathrm{material} \mathrm{points}$ are not modeled, physical methods based on shell elements have also been proposed to simulate thin muscles KHYS02. Surface bone models have been used to generate more realistic skeleton animations based on possibly cyclic graphs and moving joint centers SNTH03.

In the context of medical image analysis, subject specific models can be reconstructed using surface models based on the visible organ boundaries GRP10. Multiple images of the same subject can be exploited using sophisticated registration techniques [SKMT10]. To fit one dimensional models such as muscle action lines to subject data, post-processing is required to identify a muscle path. A widely used approach is to generate a piecewise line from the muscle cross-section centroids [ $\left.\mathrm{DLH}^{+} 90\right]$.

Volumetric representations: Although one-dimensional abstractions are sufficient in many applications, the complex muscle architecture, large muscleto-bone attachment areas, as well as muscle contacts, can only be simulated using three-dimensional models. The most widely used technique is to subdivide organs into elements and to apply continuum mechanics, typically the finite element method (FEM). Chen and Zeltzer in 1992 CZ92 modeled individual muscles as coarse linear finite elements and applied a Hill-type model to approximate their constitutive behavior. Ng-Thow-Hing in 2001 [NTH01] chose to model muscles as B-spline solids, with embedded Hill-type one dimensional elements. They proposed to simulate skin deformation, and took into account the effect of fat layer, dermal layer, and musculature, in a fi- 
nite element framework. Blemker and Delp BD05 developed and evaluated a new formulation for creating three-dimensional finite-element models that represent complex muscle geometry and the variation in moment arms across fibers within a muscle. This 3D muscle model has the advantage to represent complex muscle path motion without via point $\left[\mathrm{DLH}^{+} 90\right]$ and wrap surface BAG99, but it is computationally expensive and impractical to use in real-time applications. Teran et al $\left[\mathrm{TEB}^{+} 05\right]$ used a finite volume method (FVM) with quasi-incompressible, transversely isotropic, hyperelastic constitutive model to simulate soft tissue contraction and deformation. B-spline solids were used to model fiber directions, and the muscle activation levels were derived from key-frame animations. A framework for the construction of a 3D simulation model based on MRI data has been proposed $\left[\mathrm{ACS}^{+} 09\right]$, focusing on the hip model subject imposed bone skeletal motion. Lee, Sifakis and Terzopoulos LST09 introduced one of the most highly detailed biomechanical model of the human upper body composed of a dynamic articulated skeleton, numerous Hill-type muscle actuators which include the forcevelocity relation, and realistic finite element simulation of soft tissues and skin deformation. They used target poses and co-activation as input to compute muscle activation using inverse dynamics. The activation is then used to simulate skeleton dynamics and soft tissue deformation. The skin and underlying soft tissues were also simulated using FEM. In 2011, Stavness et al. [SLPF11] introduced an original 3D dynamic model for jaw-tongue-hyoid complex, consisting of an FEM model of the tongue, rigid jaw, and hyoid structures, Hill-type muscle actuators, constraints for bite contact and the temporomandibular joint.

Accurate musculoskeletal simulations are achievable using these detailed three dimensional representations, but at the cost of computational speed (e.g. the method of $\left[\mathrm{TEB}^{+} 05\right.$ runs at 4 minutes per frame), making these methods impractical in many real world applications. Moreover, heterogeneous models can not be easily combined or reused in different contexts, as they are not integrated into a common framework allowing for the modular design of simulation scenarios. Interpolation and extrapolation mechanisms are not available to adapt the model complexity and type of representation to application constraints. As a result, subject-specific data such as medical images are currently difficult to exploit. Without a high level multi-model description, it is tedious to produce subject specific simulations, as we need to complete the subject-specific extracted model with additional geometry and parameters not available in the data.

\subsection{Modeling Framework}

Our modeling framework is summarized in Figure 1.1 and this section presents its different modules. The main input is a set of anatomical functions 
that the user wishes to simulate (e.g. walking). Our system then produces a list of involved anatomical entities and mechanical parameters required for their simulation. A generic model is then be created, which can be complemented with subject-specific information.

\subsubsection{Symbolic description of anatomical entities}

Biomechanical simulations require an accurate anatomical description. We base our framework on symbolic knowledge of the human body, organized in a widely accepted ontology of human anatomy called Foundational Model of Anatomy (FMA) RM03. This ontology describes our body as a set of anatomical entities named classes, and connected by relations. Each class contains data named attributes. The main relations we use are :

- is_a : describes each entity as a child of another one. This relation describes the inheritance of property.

Example: The femur is a bone organ

- part_of : This relation represents each entity as a part of another one and consequently denote the inclusion.

Example: The patella is a part of knee

- inserted_on : Which is mainly used for the ligaments, muscles, tendons and ... etc. It describes the fact that one entity is inserted on another one, and then can be used to located one part of our anatomy.

Example: The vastus medialis is inserted on the femur

In order to ease the simulation of anatomical functions, we introduce a new ontology of human body functions consistent with FMA. It consists of a taxonomy of human functions, along with the new relation involved_in used to represent that an anatomical entity is involved in a given function.

Example : rectus femoris is involved in knee flexion.

In this example:

- rectus femoris is the anatomical entity (class)

- knee flexion is the biomechanical action or function (class)

- And involved in is the relation between those.

This new ontology, currently consisting of 4200 functions, was created using the Protégé 4.2 software and is stored in $O W L$ format.

\subsubsection{Reference model}

One key feature of our approach is the coupling of a universal symbolic description of anatomy with numerical data and parameters. For versatility, 
different levels of coupling are proposed. First, a collection of reference data is associated with entities in the ontology and in a second step, this reference model is enriched with custom data. The first version of our reference model includes:

- Geometry: some template bone and muscle surface meshes. For now, we have chosen to use the commercially available Zygote model as our template for its completeness. It represents an average normal subject.

- Active muscle model: one-dimensional muscle paths equipped with functional parameters. Currently, these models are adapted from Opensim $\left[\mathrm{DLH}^{+} 90\right.$, a widely used framework for musculoskeletal simulation.

- Joint and bone model: we have defined joint centres and rotation axes with respect to surface models based on available biomechanical literature. To model bone inertia, an average density is specified.

This data collection is easily extendable, and we intend to include more detailed functional information in the future, such as local fiber orientations and rheological properties. Moreover, to make our reference model both generic and parameterizable, we are collecting subject-specific data, to be averaged and integrated into a statistical atlas.

\subsubsection{Assembly}

Our framework allows the selection of the geometry, functional parameters, and even the nature of the representation (e.g., Hill-derived muscles, surface or volumetric meshes with different discretizations, etc.) as shown in the left of Figure 1.1. among a choice of built-in or external models. Once the selection is achieved, an assembly stage is necessary to connect the anatomical entities and build a fully functional model that can be automatically registered to medical data or simulated. The connections are composed of pairs of matching points (for attachments) or reference frames (for simple joint models). These features can be located with respect to others in hierarchies, as allowed by the mapping components in the SOFA framework $\left.\mathrm{FDD}^{+} 12\right]$ discussed in Section 1.4. In our prototype, these include the location of joint frames and insertion points with respect to bones, and non-rigid attachment of muscle geometry to the bones. In the future, more general interpolation functions will be implemented. For instance, the way muscle fibers will be embedded into a volumetric mesh, or the repartition of actuation amplitudes in a group of co-actuated muscles. 


\subsubsection{Registration to subject specific data}

We rely on existing registration techniques to fit our generic models to subject-specific data. Registration is a central problem in the computer graphics and image processing communities [ZF03. It proceeds by iterating two basic steps: first, the distance between the current position of the reference shape and the target is estimated; secondly, this distance is minimized by transforming the reference model. The main difficulties are to find an adequate distance measure that is as-convex-as-possible and a good parameterization of the transformation through the introduction of prior information. This introduced constraints in the deformation mechanism describe the allowed geometric excursion from the reference model. In the context of the musculoskeletal system, articulated bones can undergo large discontinuous displacements, soft tissues are highly deformable and due to shape variability in the population, geometric differences between the reference model and the subject can be relatively large. However, organ topologies, their relative positions and muscle attachment sites are stable in healthy subjects. Moreover, models must remain collision-free. We leverage this knowledge in our reconstruction framework.

The specific registration technique we use here is described in GRP10]. This technique is well suited to the musculoskeletal system because it decomposes the unknown displacement field into: (a) a global articulated motion of the bones; (b) global bone deformations from a statistical shape model generated from multiple subjects; (c) a locally smooth as-rigid-as-possible deformation field. The additional constraints are the range of motion of joints, the maximum amplitude along each statistical mode, and the stiffness of the as-rigid-as-possible deformation technique (see [GRP10 for details). Given some reference manual segmentations, a prior appearance model, containing image intensities in the neighborhood of organ surfaces, is learned. Maximizing the similarity between this appearance model and the current observed appearance in the subject images, the reference model automatically converges to the target contours, by evolving in the constrained deformation space. In our study, we use T1-weighted MR images of the complete lower limbs, acquired with an isotropic spatial resolution of $1.2 \mathrm{~mm}$. Slice-by-slice manual delineation was done once on one dataset (about 15 hours of work) to reconstruct a reference model for automatic registration (about 2 minutes of computation). Our approach is hierarchical: we first register the skeleton, then muscles. Based on comparisons with manual segmentation, the final accuracy of our model is about $1 \mathrm{~mm}$. As shown in GRP10], when target geometric models are available through manual segmentation or other sources, a similar registration procedure can be used to perform surface-to-surface registration. Structures that are not visible or outside the field of view do not undergo image-based constraints; they are automatically extrapolated based on the regularizing internal deformation constraints previously described. Our 


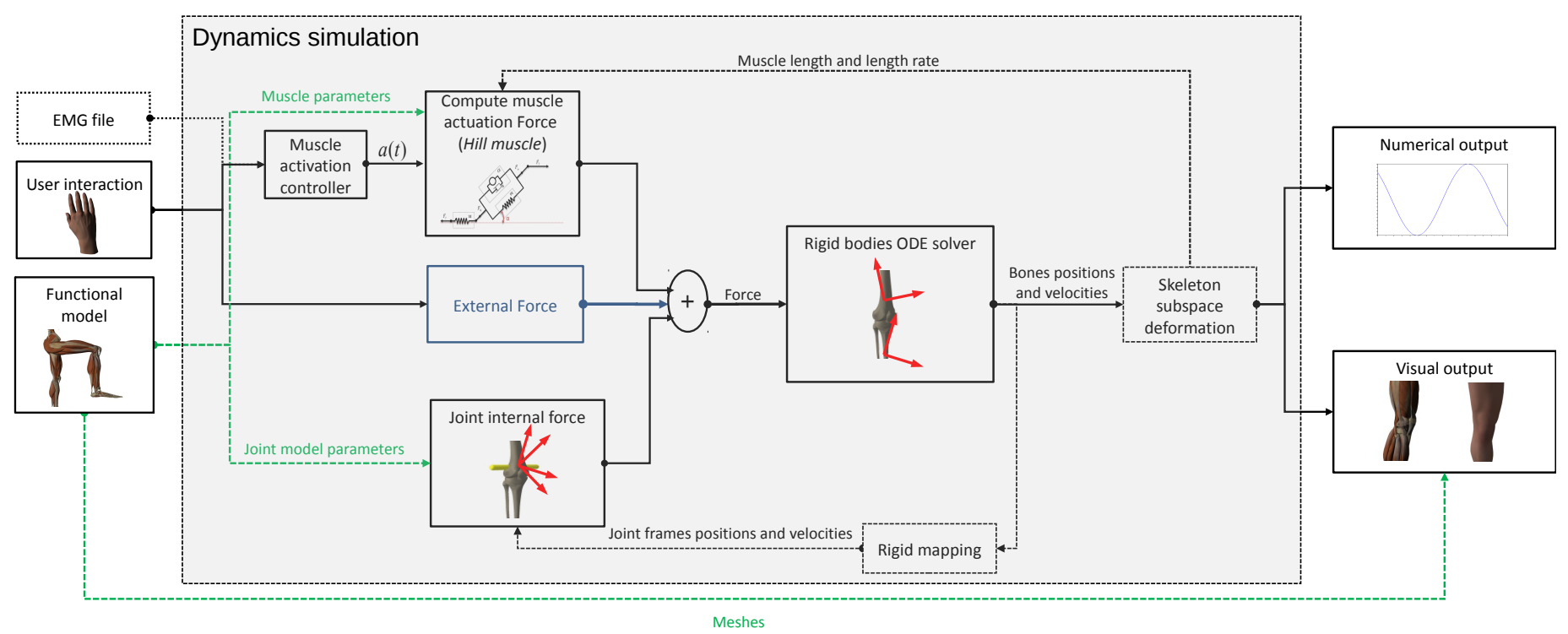

Fig. 1.2 Overview of our simulation pipeline

method has been implemented in the SOFA framework, allowing an easy and modular configuration of the registration process.

\subsection{Simulation}

We rely on a number of simulation techniques tied together using the SOFA framework. Our simulation pipeline is summarized in Figure 1.2. It combines a skeletal dynamics simulation module with Hill-type muscle actuators, and procedural methods for soft tissue deformation. The independent degrees of freedom of our simulation model are the positions and orientations of the bones, while the input parameters are the function actuation levels which can be interactively changed by the user.

Skeletal model: Each bone is represented as a rigid body equipped with a center of mass and an inertia matrix. To model joints, frames are rigidly attached to bones using a SOFA RigidMapping component. In most biomechanical frameworks $\mathrm{DLH}^{+} 90$, bone positions are expressed using reduced coordinates (the classical serial approach of robotics). In contrast with these simplified models, we use world coordinates and stiff penalty forces to enforce soft articular constraints. This allows a simpler handling of kinematic loops (such as the femur/patella/tibia complex), and it is more realistic, since slight translations are present in real biological joints. Note, however, that a 
traditional robot-like link hierarchy could be applied to the same functional model. Reference joint angle and shift limits, as well as joint stiffnesses are provided by the reference model, although they are easily customizable by the user. Stable bone dynamics with large time steps is achieved using an implicit Euler integration scheme.

Passive soft tissue model: Based on bone positions, we compute the deformation of soft tissue (e.g. skin, muscles) using a classical Skeleton subspace deformation approach [KCvO07. The transformation of a given soft tissue vertex is computed as a combination of bone transformations. In Figure 1.3 , point $P$ is associated with each bone in the reference configuration (left). During the simulation, bones are displaced as illustrated in the right of Figure 1.3. If $P$ was moving rigidly with respect to each bone, different target positions would be obtained. In linear blend skinning, the final position of $P$ is obtained by computing the weighted sum of these target positions. The influence of each bone is modulated by its weight function. Weights are computed as a function of the closest distance $d_{i}$ to each bone $i$. Here we use the function $w_{i}(P)=\tan \left(-d_{i}^{2}+\pi / 2\right)$, and subsequently normalize the weights to sum up to 1 , but other functions are possible. Other techniques such as dual quaternion blending $\mathrm{KCvO07}$ have been proposed and are applicable in our context. The vertex blending allows to preserve the approximate distances between the muscles, and therefore the fat is implicitly taken into account. The external forces acting on the soft tissue are mapped to the bones using the principle of virtual work (transpose of the Jacobian of the kinematic relationship).

Muscle actuation model: In this example, we use the one dimensional Hilltype model proposed by D.G Thelen [The03, which is a variant of the popular Delp model $\mathrm{DLH}^{+} 90$ accounting for age. Tendon and muscle elasticity is modeled using exponential stress-strain laws followed by linear functions. Active muscle forces are modeled using a stress-strain Gaussian function, based on the actuation level. Dependence on contraction velocity is also taken into account. The macroscopic model is composed of the serial assembly of the tendon elastic component and the muscle component, which is composed of the passive and active units in parallel. In summary, the macroscopic muscle model can be considered as a generalized spring that generates forces on bones at insertion points, based on its internal length, velocity and actuation level. The intrinsic muscle parameters, controlling the shape of strain-stress curves, have been adapted from the OpenSim framework $\mathrm{DLH}^{+} 90$. Specifically, they are scaled to fit the subject-specific morphology at the registration stage. In our method muscle paths are automatically deformed using skinning methods as for their external shape so there is no need to add via points and warp surfaces as done in OpenSim [SLDT07]. 

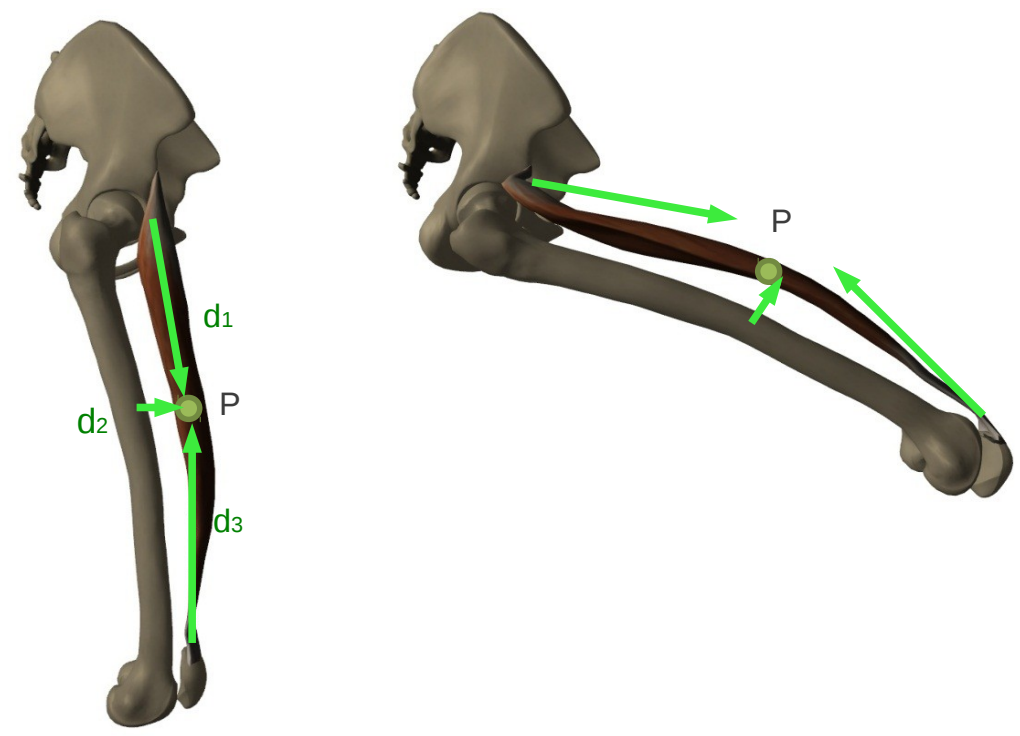

Fig. 1.3 Skinning of a point $P$ of the rectus femoris muscle by the patella, pelvis and femur bones. On the left, the original configuration used to compute the closest distance and the weights. On the right, the deformed configuration where $P$ is computed as a weighted sum of rigidly transformed positions.

\subsection{Results}

We present an example of the results obtained using the described framework. As explained, we start by selecting the functions we want to model and simulate. We choose knee flexion and extension, hip adduction, abduction, flexion and extension. Based on this, the output is a lower limb composed by 8 rigid bones linked by 7 joints, 44 muscles actuators (parameters taken from AWLD10 ), 108 muscles and 23 bone geometries as shown on the top of Figure 1.4.

Given this data as input of the simulation pipeline presented in Section 1.2 . we obtain a model allowing the realistic simulation of the targeted functions for the reference model, as shown in the bottom of Figure 1.4. The muscles can be controlled using sliders to change the levels of activation, or using an EMG file containing the time varying activation level for each muscle.

In order to simulate the same functions for a specific subject, we register the generic model to the subject-specific MRI data as shown in Figure 1.6 . The output of this registration is shown in the top of Figure 1.5. This registered model is used in the same simulation framework. The results are shown on the bottom of Figure 1.5, and a live capture of the modeling an simulation pipeline is available in the accompanying video. 


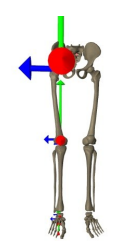

(a) Bone rigid model

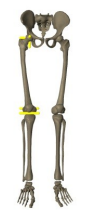

(b) Joint model

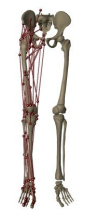

$\begin{array}{lll}\text { (c) Functional muscle model } & \text { (d) Deformation model }\end{array}$

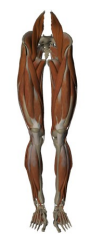

(e) Hip adduction

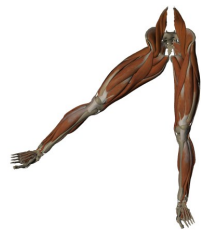

(f) Hip abduction

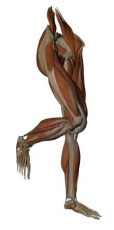

(g) Knee flexion
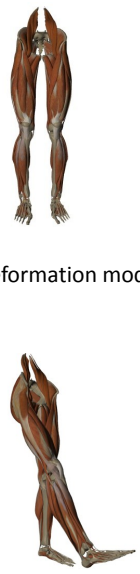

(h) Knee extension

Fig. 1.4 Simulation of the reference model. (a), (b), (c), (d) : mechanical representation of bones, joint, actuator muscle and deformable model. (e), (f), (g), (h) : hip and knee functions.

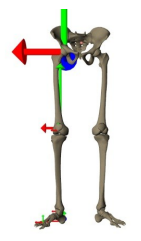

(a) Bone rigid model

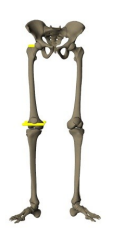

(b) Joint model

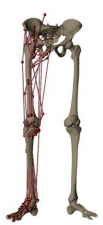

$\begin{array}{lll}\text { (c) Functional muscle model } & \text { (d) Deformation model }\end{array}$

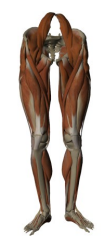

(e) Hip adduction

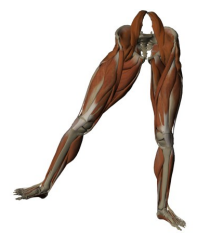

(f) Hip abduction

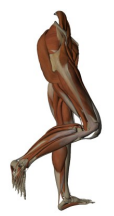

(g) Knee flexion
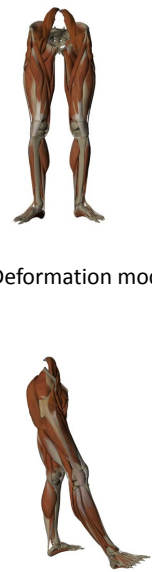

(h) Knee extension

Fig. 1.5 Simulation of the subject-specific model. (a), (b), (c), (d) : mechanical representation of bones, joint, actuator muscle and deformable model. (e), (f), (g), (h) : hip and knee functions.

These simulations run interactively at 8 frames per second on a computer with a core i7 $720 \mathrm{qm}$ at $1.60 \mathrm{GHz}$ and an NVidia quadro $1800 \mathrm{M}$ graphics board with 1 Gbyte of memory. While not achieving the same level of physical accuracy as detailed Finite Element or Finite Volume simulations, the precision of this simulation is comparable with OpenSim [SLDT07, with subject-specific geometry and improved visual realism. 


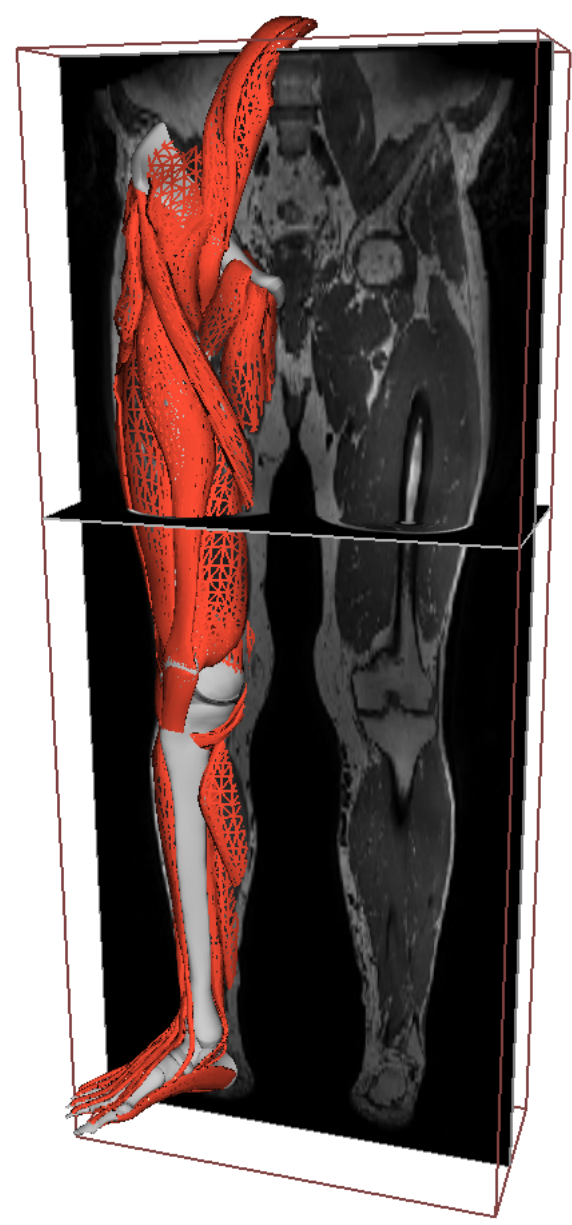

Fig. 1.6 Registration of the reference model to the subject-specific data.

\subsection{Conclusion}

We have presented a versatile modeling and simulation framework based on ontologies of human anatomy and reference models. By combining novel functional information with efficient registration techniques, our method can produce interactive, subject-specific musculoskeletal simulations. We will use this framework to generate subject-specific biomechanical models interacting with various devices as discussed in the introduction.

This novel approach of anatomical modeling raises new questions. The optimal selection of involved anatomical entities, given a user-specified musculoskeletal function, is an open question. For instance, the muscles directly involved in knee flexion (i.e., 1-ring neighbors in the ontology graph) are: 

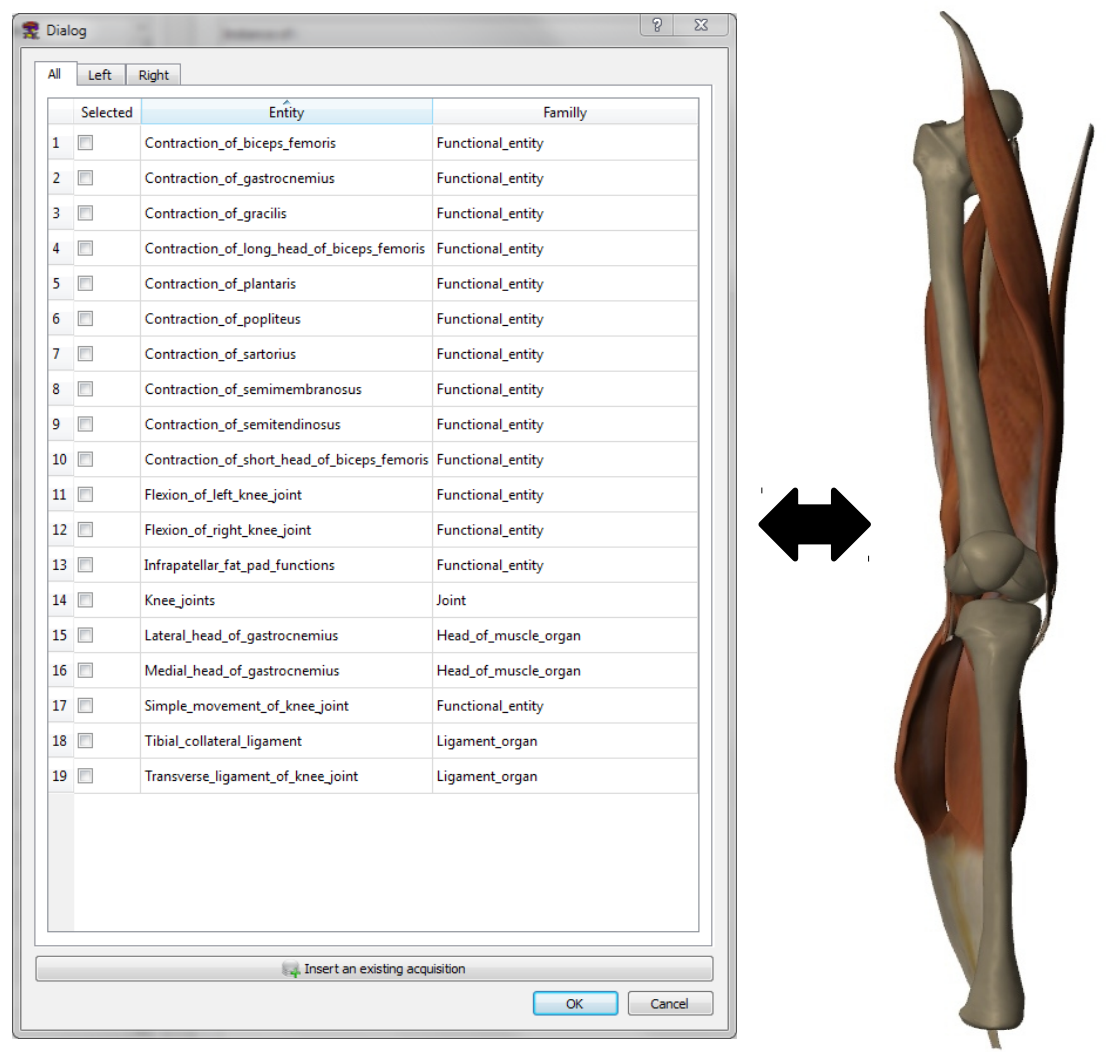

Fig. 1.7 Partial model obtained with a one-level propagation in the ontology graph from the term "knee flexion"

Biceps femoris long head, Biceps femoris short head, Gastrocnemius lateral head, Gastrocnemius medial head, Gracilis, Sartorius, Semimembranosus, Semitendinosus, as illustrated in Figure 1.7. These entities are not sufficient to create a fully functional model to simulate this function, because some features such as the presence of stabilizing and antagonistic muscles are not automatically present in the output. Some additional knowledge and input is required, such as the concatenation of functions (e.g. muscle flexion and extension) in this example. The automatic completion of models is ongoing work.

More accurate, three-dimensional mechanical models such as Finite Element meshes are necessary where accuracy is needed. The associated challenge will be to finely tune each model type and resolution (thus its computational cost) depending on the function of interest, and on the purpose of the simulation. 
We believe that by allowing the easy creation of anatomical models combining reference and specific data, our framework opens avenues for new applications of biomechanical simulation, and that it is a significant step toward an anatomical knowledge and modeling system to help users from different domains with various levels of expertise to share, customize and re-use models.

\section{References}

$\mathrm{ACS}^{+}$09. L. Assassi, C. Charbonnier, J. Schmid, , P. Volino, and N. MagnenatThalmann. From mri to anatomical simulation of the hip joint. Comput. Animat. Virtual Worlds, 20(1):53-66, January 2009.

AWLD10. E.M. Arnold, S.R. Ward, R.L. Lieber, and S.L. DELP. A model of the lower limb for analysis of human movement. Annals of Biomedical Engineering, 38(2):269-279, February 2010.

BAG99. Marcus G. PANDY Brian A. GARNER. The obstacle-set method for representing muscle paths in musculoskeletal model. 3:1-30, February 1999.

BD05. S.S. Blemker and S.L. Delp. Three-dimensional representation of complex muscle architectures and geometries. Annals of Biomedical Engineering, 33(5):661-673, 2005.

CZ92. David T. Chen and David Zeltzer. Pump it up: computer animation of a biomechanically based model of muscle using the finite element method. SIGGRAPH Comput. Graph., 26:89-98, July 1992.

$\mathrm{DLH}^{+}$90. S.L. Delp, J.P. Loan, M.G. Hoy, F.E. Zajac, E.K. Topp, and J.M. Rosen. An interactive graphics-based model of the lower extremity to study orthopaedic surgical procedures. Biomedical Engineering, IEEE Transactions on, 37(8):757 -767, aug. 1990 .

DMT07. T.T. Dao, F. Marin, and M.C. Ho Ba Tho. Ontology of the musculo-skeletal system of the lower limbs. In Engineering in Medicine and Biology Society, 2007. EMBS 2007. 29th Annual International Conference of the IEEE, pages $386-389$, aug. 2007.

$\mathrm{FDD}^{+}$12. François Faure, Christian Duriez, Hervé Delingette, Jérémie Allard, Benjamin Gilles, Stéphanie Marchesseau, Hugo Talbot, Hadrien Courtecuisse, Guillaume Bousquet, Igor Peterlik, and Stéphane Cotin. SOFA: A Multi-Model Framework for Interactive Physical Simulation In Yohan Payan, editor, Soft Tissue Biomechanical Modeling for Computer Assisted Surgery. Springer, 2012. http://www.sofa-framework.org

GGRT $^{+}$07. M. Gutierrez, A. Garcia-Rojas, D. Thalmann, F. Vexo, L. Moccozet, N. Magnenat-Thalmann, M. Mortara, and M. Spagnuolo. An ontology of virtual humans: Incorporating semantics into human shapes. Vis. Comput., 23(3):207-218, February 2007.

GRP10. Benjamin Gilles, Lionel Reveret, and Dinesh Pai. Creating and animating subject-specific anatomical models. Computer Graphics Forum, pages http://onlinelibrary.wiley.com/doi/10.1111/j.14678659.2010.01718.x/abstract, June 2010.

HG24. A. HILL. H. GASSER. The dynamics of mucular contraction. Royal Society of London Proceedings, pages 398-437, 1924.

Hil38. A. V. Hill. The heat of shortening and the dynamic constants of muscle. Proceedings of the Royal Society of London. Series B, Biological Sciences, 126(843):136-195, Oct 1938. 
KCvO07. L. Kavan, S. Collins, J. Žára, and C. O'Sullivan. Skinning with dual quaternions. In Proceedings of the 2007 symposium on Interactive $3 D$ graphics and games, I3D '07, pages 39-46. ACM, 2007.

KHYS02. K. Kahler, J. Haber, H. Yamauchi, and H.P. Seidel. Head shop: Generating animated head models with anatomical structure. ACM Symposium on Computer Animation, pages 55-64, 2002.

Lee10. Dongwoon Lee. A survey of modeling and simulation of skeletal muscle. ACM Transactions on Graphics, 28(4), Jan 2010.

LST09. S.H. Lee, E. Sifakis, and D. Terzopoulos. Comprehensive biomechanical modeling and simulation of the upper body. ACM Trans. Graph., 28:99:1-99:17, September 2009 .

MTLT88. N. Magnenat-Thalmann, R. Laperrière, and D. Thalmann. Joint-dependent local deformations for hand animation and object grasping. In Proceedings on Graphics interface '88, pages 26-33, Toronto, Ont., Canada, Canada, 1988. Canadian Information Processing Society.

NTH01. Victor Ng-Thow-Hing. Anatomically-Based Models for Physical and Geometrical Reconstruction of Humans and Other Animals. PhD thesis, 2001.

RM03. Cornelius Rosse and Jose L. V. Mejino. A reference ontology for biomedical informatics: the foundational model of anatomy. J. of Biomedical Informatics, 36:500, 2003.

Sch93. Ph.D Schutte, Lisa Margaret. Using musculoskeletal models to explore strategies for improving performance in electrical stimulation-induced leg cycle ergometry. PhD thesis, Stanford University, 1993.

SKMT10. J. Schmid, J. Kim, and N. Magnenat-Thalmann. Coupled registrationsegmentation: application to femur analysis with intra-subject multiple levels of detail mri data. In Proceedings of the 13th international conference on Medical image computing and computer-assisted intervention: Part II, MICCAI'10, pages 562-569, Berlin, Heidelberg, 2010. Springer-Verlag.

SKP08. S. Sueda, A. Kaufman, and D.K. Pai. Musculotendon simulation for hand animation. ACM Transactions on Graphics, 27(3):83:1-83:8, 2008.

SLDT07. Allison S. Arnold Peter Loan Ayman Habib Chand T. John Eran Guendelman Scott L. Delp*, Frank C. Anderson and Darryl G. Thelen. Opensim: Opensource software to create and analyze dynamic simulations of movement. IEEE Transactions on Biomedical Engineering, VOL. 54(11):1940-1950, 2007.

SLPF11. Ian Stavness, John E. Lloyd, Yohan Payan, and Sidney Fels. Coupled hardsoft tissue simulation with contact and constraints applied to jaw-tonguehyoid dynamics. International Journal for Numerical Methods in Biomedical Engineering, 27(3):367-390, 2011.

SNTH03. W. Shao and V. Ng-Thow-Hing. A generic joint component framework for realistic articulation in human characters. ACM Symposium on Interactive 3-D Graphics, pages 11-18, 2003.

TEB ${ }^{+}$05. J. Teran, S. Eftychios, S.S. Blemker, V. Ng-Thow-Hing, C. Lau, and R. Fedkiw. Creating and simulating skeletal muscle from the visible human data set. IEEE Transactions on Visualization and Computer Graphics, 11:317-328, 2005.

The03. D.G. Thelen. Adjustment of muscle mechanics model parameters to simulate dynamic contractions in older adults. ASME, 125(1):70-77, 2003.

Zaj89. F. Zajac. Muscle and tendon: properties,models,scaling,and application to biomechanics and motor control. Critical Reviews in Biomedical Engineering, 17(17):59-411, 1989.

ZF03. B. Zitová and J. Flusser. Image registration methods: a survey. Image Vision Comput., 21(11):977-1000, 2003. 